\title{
Diagnostic developers target antibiotic resistance
}

\section{Patient's immune response would tell physicians whether to prescribe.}

\section{Viviane Callier}

09 October 2015

When a patient shows up at the clinic with a cough and sore throat, there is no good way of discovering whether the infection is bacterial or viral. As a result, many clinicians prescribe antibiotics without really knowing if the drugs are necessary — a situation that contributes to the worrying rise of antibiotic resistance. But a team of researchers has collected evidence to suggest that by tracking the genetic signature of a patient's immune response to infection, they will be able to distinguish bacterial infections from other sources of illness.

The team, led by clinician-scientists Ephraim Tsalik, Geoffrey Ginsburg and Chris Woods at the Duke Center for Applied Genomics and Precision Medicine in Durham, North Carolina, have been developing a diagnostic tool that tracks the body's response to infection rather than the presence of a particular pathogen. Tsalik plans to discuss the team's research on 16 October, at a workshop at the US Food and Drug Administration (FDA) in Silver Spring, Maryland.

Rapid-detection tests or throat cultures that are used to detect streptococcus and other common bacteria can miss more than $50 \%$ of infections and are plagued with false positives. The resulting overuse of antibiotics contributes to antibiotic resistance, a growing public-health threat. The US Centers for Disease Control and Prevention estimates that antibiotic-resistant infections kill at least 23,000 people each year in the United States. And in India, the percentage of Klebsiella pneumoniae infections that are resistant to powerful carbapenem drugs rose from $29 \%$ in 2008 to $57 \%$ in 2014 .

The Duke team's hypothesis - that specific changes in gene activity might betray the type of infection that the body is responding to - is a reasonable one, says David Relman, a microbiologist at Stanford University in California. "It's not terribly likely that the host response alone will be sufficient for identifying the pathogen, but it could be used to predict a class of illness and help clinicians decide on a general treatment approach," Relman says.

\section{Clinical challenges}

Over six years, the Duke team collected blood samples from more than 300 patients who came to the hospital with symptoms consistent with a viral or bacterial infection. Retrospectively, the researchers determined which patients actually had which kind of infection and analysed the genes expressed in the immune cells from the blood samples.

"There are a number of groups working in this area that just a few years back were laughing at us," notes Woods. "Most people didn't believe this was a useful approach."

But the team was able to show that the gene-expression patterns reliably distinguished between bacterial and viral or non-infectious illness. "The signal was terrific," Ginsburg says.

In the last decade, improvements in methods for measuring the expression of many genes at once, accompanied by the development of new statistical methods to analyse the data, were crucial to the team's success.

The real challenge now, the researchers say, is to transform this research into a clinical tool. The focus of the FDA workshop, explains the FDA's infectious-disease specialist Steven Gitterman, will be to consider the performance standards to which these new technologies should be held and to develop appropriate clinical-trial designs to evaluate their safety and accuracy. 
to show that different populations around the world all respond similarly to bacterial infections.

The technology also needs to deliver information in a clinically relevant time frame, the researchers say. The team is working with industry partners to develop a platform that can reduce the time required to measure the genes of interest from days to an hour or less. If that goal can be achieved, says Woods, "that changes how we deal with patients altogether".

Nature | doi:10.1038/nature.2015.18512 